\title{
Multi-Operator Fairness in Transparent RAN Sharing by Soft-Partition With Blocking and Dropping Mechanism
}

\author{
Hsu-Tung Chien, Ying-Dar Lin, Fellow, IEEE, Hsien-Wen Chang, and Chia-Lin Lai
}

\begin{abstract}
Radio access network (RAN) sharing has attracted significant attention from telecom operators as a means of accommodating data surges. However, current mechanisms for RAN sharing ignore the fairness issue among operators, and hence the RAN may be under- or over-utilized. Furthermore, the fairness among different operators cannot be guaranteed, since the RAN resources are distributed on a first come, first served basis. Accordingly, the present study proposes a "soft-partition with blocking and dropping" (SBD) mechanism that offers inter-operator fairness using a "soft-partition" approach. In particular, the operator subscribers are permitted to overuse the resources specified in the predefined service-level-agreement when the shared RAN is under-utilized, but are blocked (or even dropped) when the RAN is over-utilized. The simulation results show that SBD achieves an inter-operator fairness of 0.997 , which is higher than that of both a hard-partition approach (0.98) and a no-partition approach (0.6) while maintaining a shared RAN utilization rate of $98 \%$. Furthermore, SBD reduces the blocking rate from $35 \%$ (hard partition approach) to almost $0 \%$, whereas controlling the dropping rate at $5 \%$. Notably, the dropping rate can be reduced to almost $0 \%$ using a newly proposed bandwidth scale down procedure.
\end{abstract}

Index Terms-Radio access network (RAN), RAN sharing, soft partition, fairness, utilization, blocking rate, dropping rate.

\section{INTRODUCTION}

$\mathbf{R}$ AN sharing is an attractive solution for telecom operators seeking to address upcoming data surges with minimum investment in CAPEX and OPEX [1], [2], [3]. Transparent RAN sharing, in which RAN sharing is achieved through an intermediate mechanism between the base station (BS) and multiple core networks (CNs), is particularly attractive to op-

This work was supported in part by H2020 collaborative Europe/Taiwan research project 5G-CORAL under Grant 761586, and in part by the Ministry of Science and Technology, Taiwan under Contract MOST 106-2218E-009-018. The review of this paper was coordinated by Prof. Y. Qian. (Corresponding author: Hsu-Tung Chien.)

H.-T. Chien is with the Department of Computer Science, National Chiao Tung University, Hsinchu 300, Taiwan, and also with the Information and Communications Research Laboratories, Industrial Technology Research Institute, Hsinchu 31057, Taiwan (e-mail: hsutung@ cs.nctu.edu.tw).

Y.-D. Lin is with the Department of Computer Science, National Chiao Tung University, Hsinchu 300, Taiwan (e-mail: ydlin@cs.nctu.edu.tw).

H.-W. Chang and C.-L. Lai are with the Information and Communications Research Laboratories, Industrial Technology Research Institute, Hsinchu 31057 , Taiwan (e-mail: seanchang @itri.org.tw; Chia-LinLai@itri.org.tw). erators since: (1) it is easier and more cost effective to set up RAN sharing using existing infrastructure; and (2) RAN sharing can be managed by a third-party to assure independence and fairness among different operators [4], [5]. Accordingly, the current authors previously proposed a transparent RAN sharing mechanism designated as RAN Proxy (RANP) [6]. The emulation results confirmed the effectiveness of the proposed mechanism in ensuring operator fairness in a multi-operator RAN environment.

However, to improve the practicality of RAN sharing, the fairness and efficiency issues require further consideration. RAN resource is limited; particularly the capacity of the BS in terms of the amount of served user equipment (UE) and available bandwidth. For example, in LTE, the number of RRC-connected [7] users for a macro cell is more than 1000, but the number drops to just 50-100 for a typical 5G small cell [8], [9] due to its smaller coverage. Furthermore, the LTE bandwidth is frequently constrained by bottlenecks in the backhaul network. Although LTE provides sophisticated mechanisms for individual operators to coordinate the UE and bandwidth within their own networks, its support of coordination among different operators sharing the same BS is rather poor. Given the absence of a coordination mechanism, and assuming that new UE are simply allowed to attach to the shared BS regardless of their operator until the BS reaches its capacity, very large operators with large subscriber bases may consume most of the BS resources, and hence the UE of smaller operators may suffer an unacceptable quality of experience (QoE). Conversely, if the BS resources are strictly reserved for each operator in accordance with a predefined interoperator agreement, the QoE of the UE belonging to each operator is guaranteed, but the BS utilization rate is degraded if any operator fails to use all of the resources reserved for it. Thus, both the traffic and the revenue for the third party providing the RAN sharing service are inevitably reduced [2]. In other words, the success of RAN sharing depends on achieving a satisfactory trade-off between the (potentially) competing requirements of different operators and their UE.

Accordingly, this paper proposes a "Soft-partition with Blocking and Dropping" (SBD) mechanism for controlling the fairness among different operators based on a "soft-partition" concept, in which the telecom operators are permitted to use more resources than originally agreed in the inter-operator agreement when additional resources become available at the BS. However, if the network subsequently becomes fully loaded, 


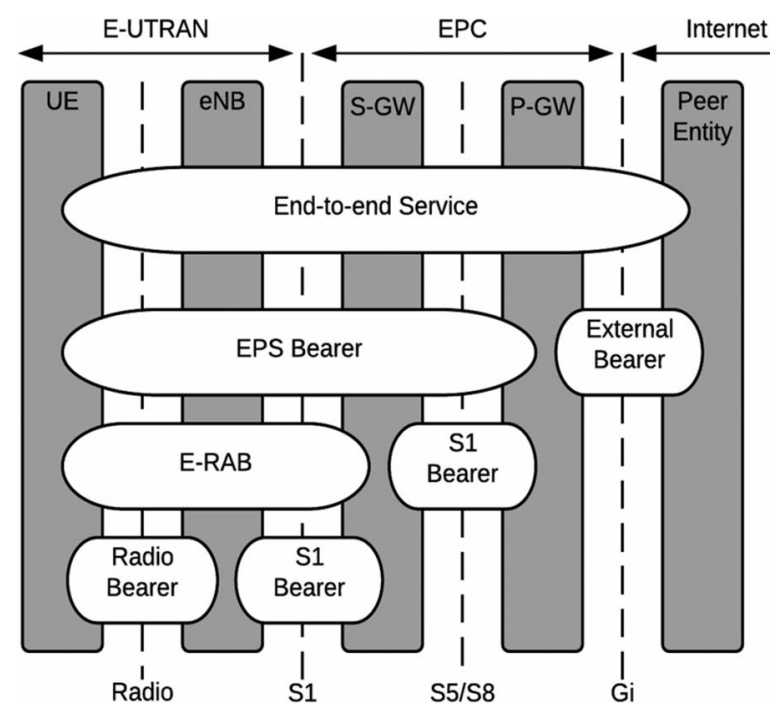

Fig. 1. LTE bearer overview.

any over-using operators are prompted to return the overused resource in accordance with a further predefined agreement. Thus, the proposed mechanism both increases the utilization of the RAN resources and ensures that the requirements of each operator are satisfied. Notably, the SBD mechanism is designed in such a way that the blocking and dropping rates of each operator are maintained at an acceptable level at all times.

The remainder of this paper is organized as follows. Section II provides the background to the present study and describes the related works. Sections III and IV formulate the considered problem and explain the detailed design of the proposed SBD mechanism, respectively. Section V presents and discusses the numerical results obtained for the fairness among multiple operators, the utilization rate of the shared BS, and the blocking/dropping rates of the operator resource requests. Finally, Section VI provides some brief concluding remarks and indicates the intended direction of future research.

\section{BACKGROUND AND RELATED WORKS}

\section{A. Intra-Operator Control: LTE QoS}

The QoS mechanism of LTE [10]-[12] is based on an Evolved Packet System (EPS) bearer [13] method. EPS bearers comprise Evolved Radio Access Bearers (E-RABs) and S1 bearers, as shown in Fig. 1 [10], and serve to link the UE and their Packet Data Network gateway (P-GW). Telecom operators are aware of the status of the EPS bearers and manage them by setting up rules in a traffic flow template (TFT) [14]. As shown in Fig. 2, EPS bearers can be further categorized as either default bearers or dedicated bearers. Default bearers are created whenever a UE attaches successfully to the $\mathrm{CN}$ and is classified as Non-Guaranteed Bit Rate (non-GBR), i.e., the network does not guarantee resources for the associated traffic. Dedicated bearers, on the other hand, are formed when required by special SLA and may be classified as either GBR or non-GBR, where in the former case, the network guarantees resources to the traffic in accordance with the TFT.

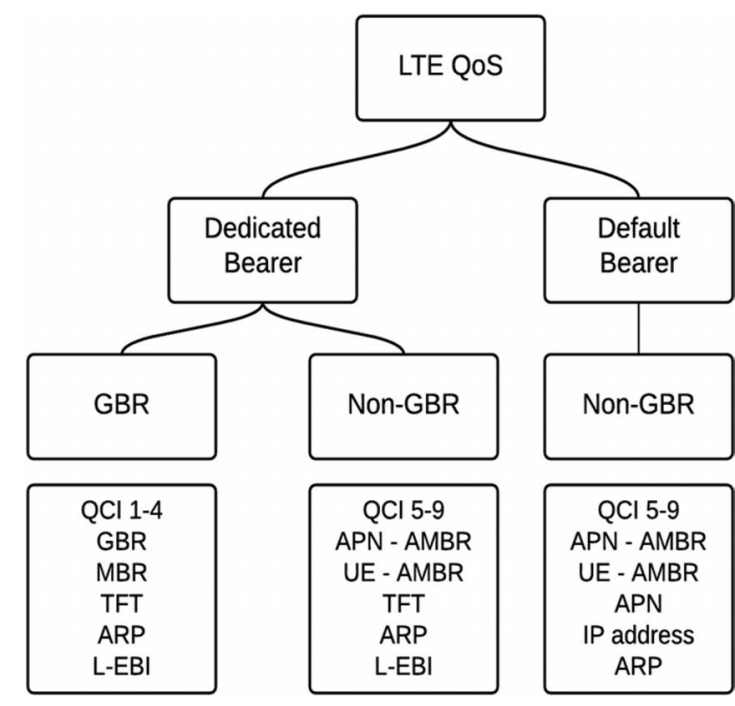

Fig. 2. Attributes of LTE bearer.

\section{B. Inter-Operator Control: Related Works}

In 2004, Nokia [15] proposed a solution for radio resource management among multiple operators in $3 \mathrm{G}$ roaming scenarios using a Control-Plane-based Hard Partition approach. Qualcomm [16] presented a similar strategy based on both Control-Plane and Data-Plane methods. In the latter method, a mechanism referred to as "Erase Packet" was used to release radio resources by detecting and deleting packets with the longest queue time whenever the BS ran out of resources. Having reorganized the resources, the Control-Plane mechanism was used to decide whether or not to accept new requests. The authors in [17] proposed a soft partition method based on an extended Network Virtualization Substrate (NVS) slice scheduler and a two-step Access Control (AC) scheme in order to enhance the access control of the bearers.

Table I compares the main attributes of the schemes presented in [15]-[17] with those of the SBD mechanism proposed in the present study. As shown, among the four schemes, SBD and the method proposed in [17] both apply a soft partitioning approach for BS resource utilization. However, only SBD has the overused resource returning mechanism. Moreover, only SBD considers the UE admission control issue caused by the high density and low capacity of the small cells in 5G networks. Finally, only SBD provides a bandwidth scale-down solution to reduce the blocking and dropping rates.

\section{PROBLEM ForMULATION}

This section formulates the BS resource allocation problem considered in the present study. For convenience, all of the related notations are defined in Table II below. In implementing the proposed SBD mechanism, an Inter-Operator Policy is used to define the agreement among the mobile network operators regarding resource coordination, and is subject to the following constraint

$$
\sum_{i=1}^{n} p_{i}^{\text {inter_ue }}=\sum_{i=1}^{n} p_{i}^{\text {inter_bw }}=1
$$


TABLE I

COMPARISON OF RELATED WORKS

\begin{tabular}{|c|c|c|c|c|c|c|}
\hline Papers / Patents & Location & $\begin{array}{l}\text { Data / Control } \\
\text { Plane } \\
\end{array}$ & $\begin{array}{l}\text { Hard / Soft } \\
\text { Partition }\end{array}$ & $\begin{array}{l}\text { UE Admission } \\
\text { Control }\end{array}$ & $\begin{array}{c}\text { Bearer Admission } \\
\text { Control }\end{array}$ & Method \\
\hline Roaming Based [15] & Core & $\mathrm{CP}$ & Hard & No & No & - CN Control \\
\hline $\begin{array}{c}\text { Asymmetric RAN Resource } \\
\text { Allocation [16] }\end{array}$ & Base Station & $\mathrm{CP} / \mathrm{DP}$ & Hard & No & Yes & $\begin{array}{l}\text { - Blocking } \\
\text { - Weighted Queueing } \\
\text { - Erase Packet } \\
\end{array}$ \\
\hline $\begin{array}{c}\text { Active LTE RAN Sharing } \\
\text { with Partial Resource } \\
\text { Reservation [17] }\end{array}$ & $\begin{array}{c}\text { ANS-Gateway } \\
\text { and } \\
\text { Base Station } \\
\end{array}$ & $\mathrm{CP} / \mathrm{DP}$ & Soft & No & Yes & $\begin{array}{l}\text { - Modified NVS slice scheduler } \\
\text { - A two-step AC scheme }\end{array}$ \\
\hline The proposed SBD & RAN Proxy & $\mathrm{CP}$ & Soft & Yes & Yes & $\begin{array}{l}\text { - Blocking and Dropping } \\
\text { - Temporarily Over Quota } \\
\text { - Scale-Down }\end{array}$ \\
\hline
\end{tabular}

TABLE II

NOTATIONS LIST

\begin{tabular}{|c|l|}
\hline Notation & \multicolumn{1}{|c|}{ Meaning } \\
\hline$P^{\text {inter_ue }}$ & Percentage of UE assigned to $i$-th operator \\
\hline$P^{\text {inter_bw }}$ & $\begin{array}{l}\text { Percentage of bandwidth assigned to } i \text {-th } \\
\text { operator }\end{array}$ \\
\hline$F^{u e}$ & System fairness in terms of number of UE \\
\hline$F^{b w}$ & $\begin{array}{l}\text { System fairness in terms of number of } \\
\text { bandwidth }\end{array}$ \\
\hline$B S^{\text {limit_ue }}$ & Maximum number of served UE by BS \\
\hline$B S^{\text {limit_bw }}$ & Maximum bandwidth of BS \\
\hline$B S^{\text {free_ue }}$ & Number of additional UE that BS can serve \\
\hline$B S^{\text {free_bw }}$ & $\begin{array}{l}\text { Amount of additional bandwidth that BS can } \\
\text { serve }\end{array}$ \\
\hline$S_{i}^{\text {ue }}$ & Number of in-use UE of $i$-th operator \\
\hline$S_{i}^{b w}$ & Quantities of in-use bandwidth of $i$-th operator \\
\hline$U^{u e}$ & $\begin{array}{l}\text { Utilization rate of shared BS in terms of } \\
\text { number of served UE }\end{array}$ \\
\hline$U^{b w}$ & $\begin{array}{l}\text { Utilization rate of BS in terms of bandwidth } \\
\text { usage }\end{array}$ \\
\hline$R_{i}^{\text {block_ue }}$ & UE blocking rate of $i$-th operator \\
\hline$R_{i}^{\text {block_bw }}$ & Bandwidth blocking rate of $i$-th operator \\
\hline$R_{i}^{\text {drop_ue }}$ & UE dropping rate of $i$-th operator \\
\hline$R_{i}^{\text {drop_bw }}$ & Bandwidth dropping rate of $i$-th operator \\
\hline
\end{tabular}

where $P^{\text {inter_ue }}=\left\{p_{i}^{\text {inter_ue }}\right\}$ and $P^{\text {inter_ue }}=\left\{p_{i}^{\text {inter_bw }}\right\}$ are the percentage of UE and bandwidth resource, respectively, allocated to the i-th operator.

Given $B S^{\text {limit_ue }}$, i.e., the maximum number of served UE for the BS, the i-th operator's quota for the number of served UE is calculated in accordance with (1) as $p_{i}^{\text {inter_ue }} \times B S^{\text {limit_ue }}$. Similarly, given $B S^{\text {limit_bw }}$, i.e., the maximum bandwidth of the BS, the i-th operator's quota for the bandwidth usage is calculated as $p_{i}^{\text {inter_bw }} \times B S^{\text {limit }}$ bw .

The fairness of the RAN system in terms of the number of served UE and bandwidth usage, respectively, can be defined as

$$
F^{u e}=\prod_{i=1}^{n} \frac{S_{i}^{u e}}{P_{i}^{\text {inter_ue }} \times B S^{\text {limit_ue }}},
$$

and

$$
F^{b w}=\prod_{i=1}^{n} \frac{S_{i}^{b w}}{P_{i}^{\text {inter_bw }} \times B S^{\text {limit_bw }}}
$$

where $S_{i}^{u e}$ and $S_{i}^{b w}$ are the quantities of in-use UE and bandwidth of the i-th operator, respectively. According to (2) and (3), as $F^{u e}$ (or $F^{b w}$ ) approaches 1 , the actual resource assignment of the i-th operator approaches that defined in the Inter-Operator Policy, i.e., the fairness of the system improves. Consider a simple system with two operators, and assume that $p_{1}^{\text {inter_ue }}$ and $p_{2}^{\text {inter_ue }}$ are both set to $50 \%$, while BSlimit_ue is given as 100 . Suppose further that the system is fully used. If $S_{1}^{u e}$ and $S_{2}^{u e}$ are both equal to 50 , then $\sum_{i=1}^{n} p_{i}^{\text {inter }-u e}$ is obtained from (1) as 1 . In other words, the system is perfectly fair.

The utilization of the shared BS can be defined in terms of the number of served UE and the bandwidth usage as

$$
U^{u e}=\frac{\sum_{i=1}^{n} S_{i}^{u e}}{B S^{\text {limit_ue }}} \times 100 \% \leq 1,
$$

and

$$
U^{b w}=\frac{\sum_{i=1}^{n} S_{i}^{b w}}{B S^{\text {limit } \_b w}} \times 100 \% \leq 1,
$$

respectively, and provides a useful measure for evaluating the resource utilization efficiency of different resource allocation methods.

As suggested by its name, "Soft-partition with Blocking and Dropping", i.e., the SBD protocol proposed in this study blocks a certain portion of the new resource requests and drops a certain portion of the in-use resources. As a result, the QoE of the UE may be adversely affected. In practice, the impact of SBD can be evaluated by the blocking rates of the UE Attach Requests and bandwidth requests, which are defined respectively as

$$
R_{i}^{\text {block } \_ \text {e }}=\frac{\text { Blocked UE }}{\text { Total Initial Attach Requests }} \times 100 \%,
$$

and

$$
R_{i}^{\text {block_bw }}=\frac{\text { Blocked Bandwidth }}{\text { Total Bandwidth Requests }} \times 100 \% .
$$

The impact can also be evaluated by the dropping rates of the in-use UE and bandwidth, which are defined respectively as

$$
R_{i}^{\text {drop_ue }}=\frac{\text { Drop UE }}{\text { Total Initial Attach Requests }} \times 100 \%,
$$

and

$$
R_{i}^{\text {drop_bw }}=\frac{\text { Drop Bandwidth }}{\text { Total Bandwidth Requests }} \times 100 \% .
$$




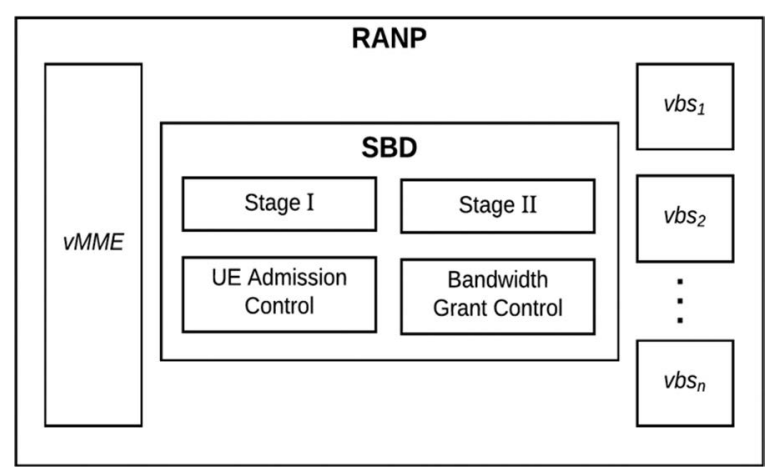

Fig. 3. Soft-partition with blocking and dropping design.

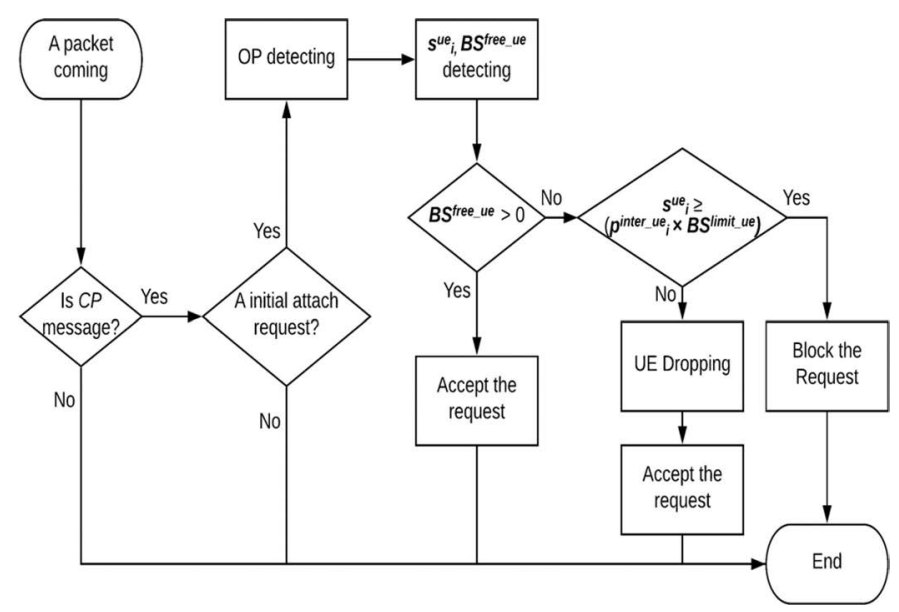

Fig. 4. UE admission control procedure.

\section{Proposed Blocking and Dropping CoORdinator}

As shown in Fig. 3, the proposed SBD protocol is integrated with the RAN Proxy (RANP) transparent RAN sharing mechanism proposed by the present group in [6], in which the communications between the $\mathrm{BS}$ and $\mathrm{CN}$ are handled by a single virtual Mobility Management Entity (vMME) module and multiple virtual base station (vBS) modules. SBD provides a twostage control of the two types of fundamental resource in a shared BS, namely the served UE and the bandwidth. In the first stage, designated as UE Admission Control, SBD is triggered by a UE Initial Attach request, and decides whether to accept or block the request, or whether to drop some in-service UE whose operators have overused their predefined number of UE. In the second stage, designated as Bandwidth Grant Control, SBD detects the E-RAB Request and E-RAB Modify messages [18] and decides whether to accept or block new bearers, or drop some existing bearers whose operators have overused their predefined guaranteed bandwidth.

\section{A. UE Admission Control}

Fig. 4 shows the main steps in the SBD UE Admission Control procedure. When a new packet arrives, SBD inspects it and identifies its type. If it is an Initial Attach request, SBD further identifies the UE's operator and retrieves the current status of

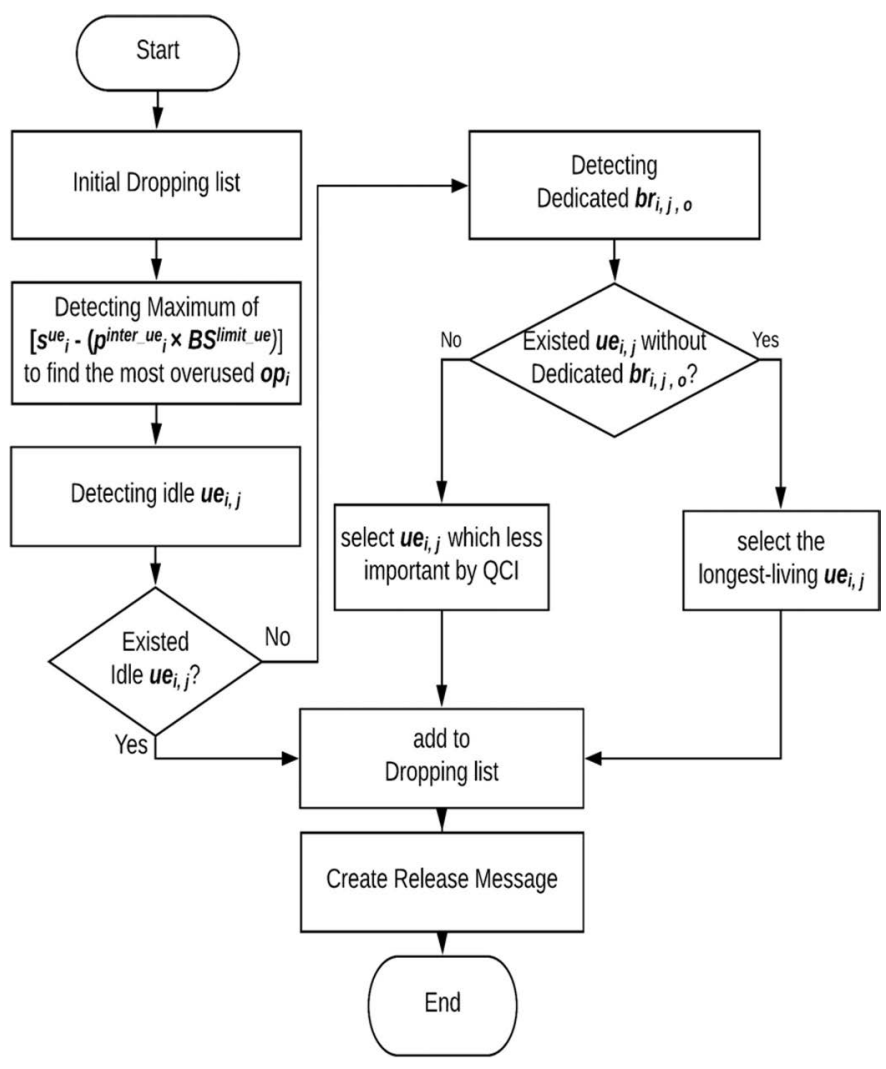

Fig. 5. UE dropping procedure.

$B S^{\text {free-ue }}$ (i.e., the number of additional UE that the BS can serve) and $S_{i}^{u e}$ (i.e., the number of in-service UE of the operator). Given this information, SBD decides whether to accept or block the Initial Attach request by checking if $S_{i}^{u e}$ is larger than the operator's quota. If $B S^{\text {free } u e}$ is greater than zero, $\mathrm{SBD}$ accepts all the requests irrespective of the value of $S_{i}^{u e}$. However, if $B S^{\text {free-ue }}$ is equal to zero, and $S_{i}^{u e}$ is larger than the operator's quota, SBD blocks the request. Conversely, if $S_{i}^{u e}$ is smaller than the operator's quota, SBD initiates the UE Dropping procedure (Fig. 5) to retrieve overused resource from the other operators such that it can accept the request. To facilitate the UE Dropping procedure, SBD maintains a dropping list from which one candidate is selected each time. In selecting a candidate for dropping, SBD first looks for the operator with the greatest overuse of UE, i.e.,

$$
\max _{i} S_{i}^{u e}-\left(p_{i}^{\text {inter_ue }} \times B S^{\text {limir_ue }}\right) .
$$

Once SBD identifies a suitable operator, it selects an idle UE from among the operator's subscribers. If an idle UE cannot be found, SBD detects the status of all the UE bearers $\left(b r_{i, j, o}\right)$ and searches for a UE with no extend dedicated bearers from $b r_{i, j, o}$, (i.e., the UE has only a default bearer). If SBD detects more than one such UE, it selects the UE with the longest connection time and adds it to the dropping list. If every UE has extend dedicated bearers, the UE with the lowest QoS Class Identifier (QCI) priority is selected and added to the dropping list. 


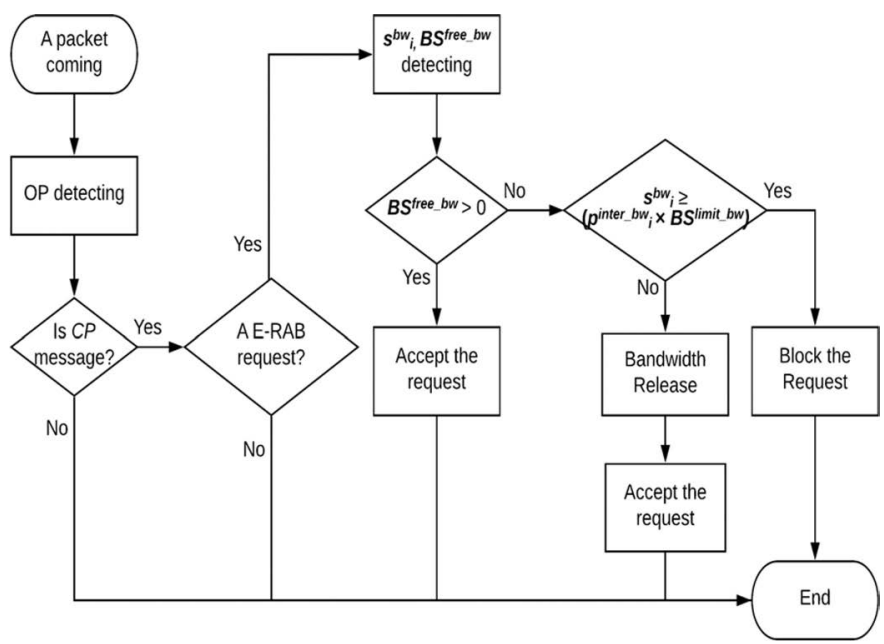

Fig. 6. Bandwidth grant control procedure.

\section{B. Bandwidth Grant Control}

Fig. 6 shows the details of the SBD Bandwidth Grant Control stage. When a packet arrives, SBD inspects it to identify its operator and ascertains whether it is an E-RAB Request or an E-RAB Modify message. SBD then retrieves the current status of $B S^{\text {free_bw }}$ (i.e., the bandwidth available at the shared BS for allocation) and $S_{i}^{b w}$ (i.e., the bandwidth allocated to the identified operator), and decides whether to accept or block the request. If $B S^{\text {free } b w}$ (i.e., the number of additional UE the BS can serve) is greater than zero, SBD accepts the request. If $B S^{f r e e} b w$ equals zero, and $S_{i}^{b w}$ is larger than the operator's quota, SBD blocks the request. Conversely, if $B S^{\text {free } b w}$ equals zero, but $S_{i}^{b w}$ is less than the operator's quota, SBD initiates the bandwidth release procedure (Fig. 7) to trigger the other operators to return overused resource such that the request can be accepted.

As for the UE Dropping procedure, SBD also maintains a dropping list for the Bandwidth Release procedure. However, the received bandwidth requests may have different sizes. Thus, in contrast to the one-pass approach used in the UE dropping procedure, the Bandwidth Release procedure iterates until a sufficient amount of resource has been released. Briefly, SBD first identifies the operator with the greatest amount of bandwidth overuse, i.e.,

$$
\max _{i} S_{i}^{b w}-\left(P_{i}^{\text {inter } b w} \times B S^{\text {limit } b w}\right) .
$$

Once an operator has been found, SBD searches for a UE with extend dedicated bearers. If every UE has extend dedicated bearers, SBD selects the UE with the lowest QCI priority and adds it to the dropping list. If several UE have the same QCI, SBD adds the UE with the longest connection time to the list. If none of the UE have dedicated bearers, SBD detects the status of all the UE bearers $\left(b r_{i, j, o}\right)$ and identifies the UE with the longest connection time. Finally, SBD calculates the amount of resource released by the chosen UE. If the released resource is insufficient to satisfy the requirements of the new request, the procedure is repeated iteratively until sufficient resource has been returned.

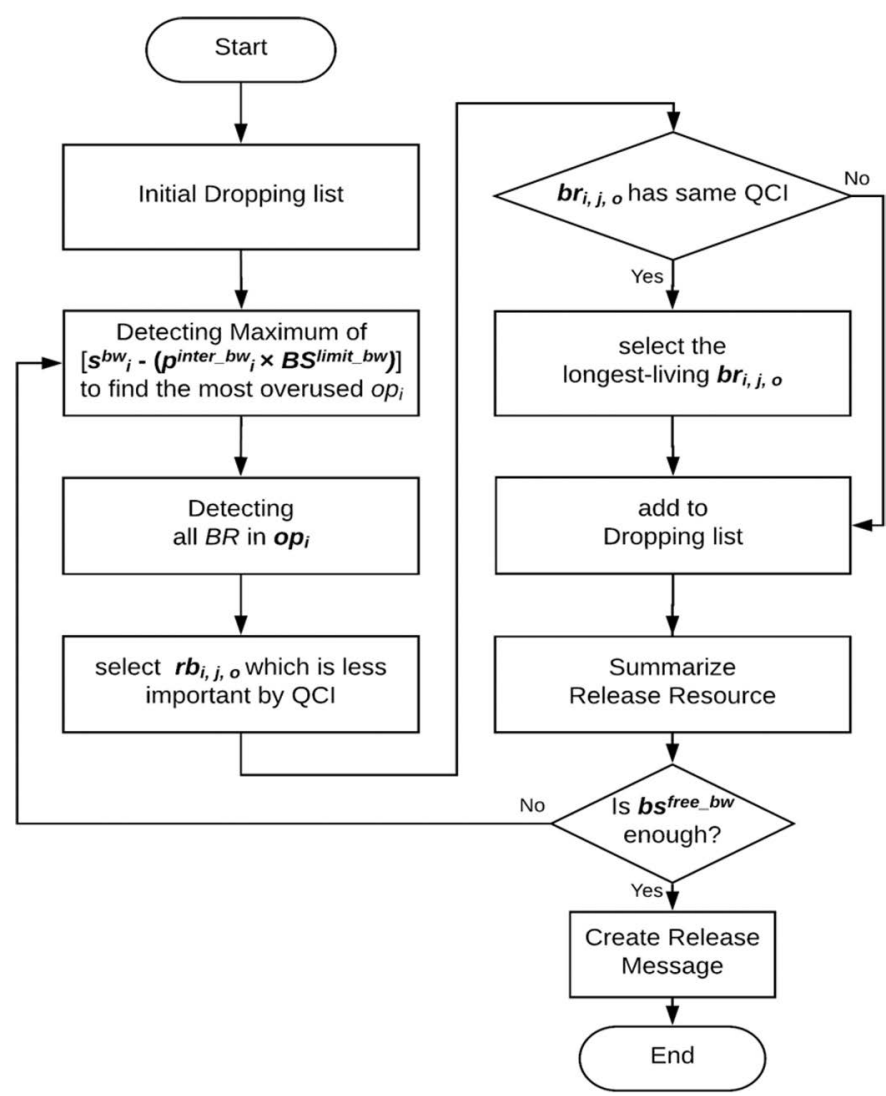

Fig. 7. Bandwidth release procedure: blocking and dropping.

When a bearer is dropped, all of the bandwidth allocated to it is released. Hence, an over dropping phenomenon may occur. As a result, the Bandwidth Release procedure described above may incur a high dropping rate. Accordingly, SBD implements an additional Bandwidth Scale-Down procedure prior to the Bandwidth Release stage described above. The premise for the proposed procedure is that the $\mathrm{CN}$ can be controlled (at least partly) by third parties through a mechanism such an operatorauthorized API (application programming interface), and hence RANP can send E-RAB messages such as E-RAB Modify to modify the bandwidth of the bearers. The main steps in the Bandwidth Scale-Down procedure are illustrated in Fig. 8. As shown, SBD first uses (11) to identify the operator with the greatest amount of bandwidth overuse and then scales down the bandwidth of the associated Non-GBR bearers by a predefined amount, $s c \%$. If the released resource is insufficient to meet the new bandwidth request, the procedure scales down the bandwidth of the dedicated bearers. In particular, SBD detects all the dedicated bearers of the operator's UE. If a single dedicated bearer is found, SBD selects it and scales its bandwidth down to its guaranteed bandwidth. Conversely, if multiple UE exist, SBD selects the UE with the lowest QCI priority and then scales down its bandwidth. In the event that all of the UE have the same QCI priority, SBD selects the UE with the longest connection time. Finally, if SBD finds no UE with dedicated bearers, or no dedicated bearers have additional bandwidth which can be scaled down, the scale down procedure is terminated and SBD moves to the blocking and dropping procedures described above. 


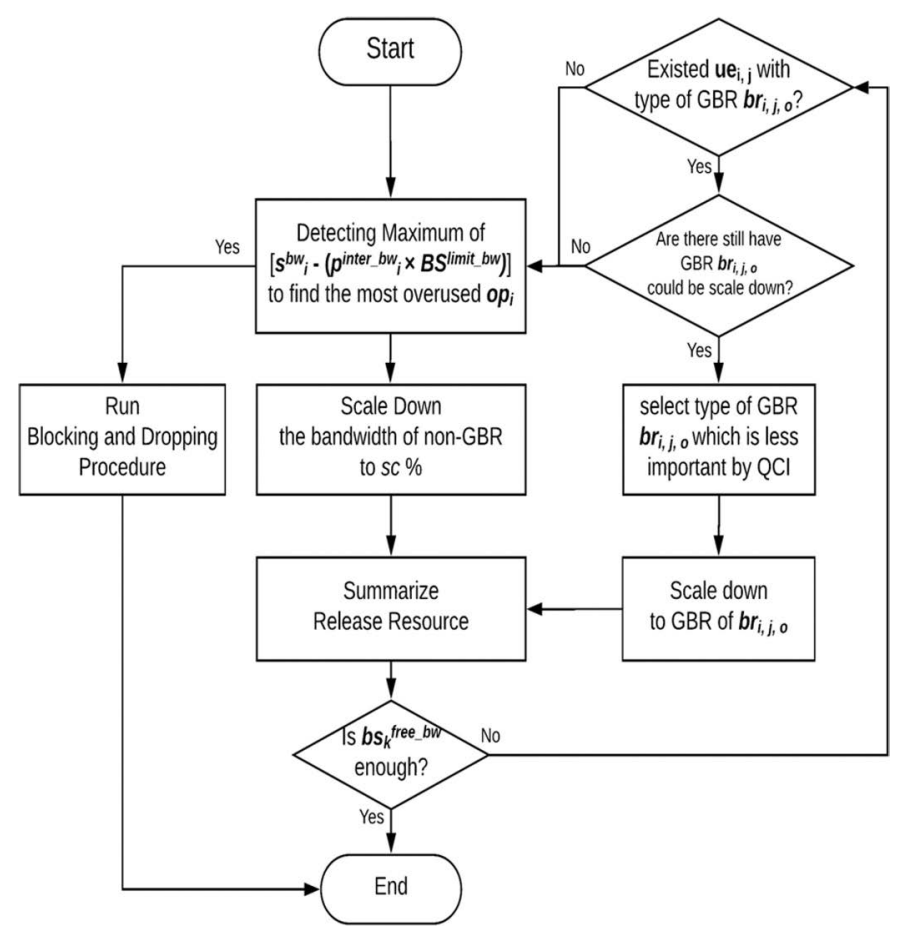

Fig. 8. Bandwidth release procedure with bandwidth scale-down.

\section{NuMERICAL RESULTS}

\section{A. Simulation Setup}

For simplicity, the simulations considered a network with just two operators. Moreover, the operators were assumed to share the resources evenly between them, i.e.,

$$
p_{i}^{\text {inter-ue }}=p_{i}^{\text {inter_bw }}=0.5, \text { where } \mathrm{i}=1,2 .
$$

In implementing the simulations, the maximum number of served UE and amount of bandwidth available for allocation at the shared BS were set as $100 \mathrm{UE}$ [8] and $100 \mathrm{Mbps}$ (refer to LTE CAT. 3), respectively. Furthermore, in accordance with (9), the UE and bandwidth quotas for each operator were set as $50 \mathrm{UE}$ and $50 \mathrm{Mbps}$. In addition, the arriving UE and bandwidth requests from operator- $i$ were assumed to follow Poisson distribution with arrival rates of $\lambda_{i}{ }^{u e}$ and $\lambda_{i}^{b w}$, respectively. The fairness evaluation experiments assumed a full-loading condition. By contrast, the utilization and blocking/dropping rate evaluation experiments assumed that the system was not fully loaded. Every simulation considered a network operation time of 500 minutes, during which time in-use resource (UE or bandwidth) was released whenever the corresponding in-use time reached 250 minutes.

\section{B. Results}

The effectiveness of SBD was evaluated in terms of three factors, namely: (1) the resource fairness between the two operators; (2) the total resource utilization of the shared BS; and (3) the blocking and dropping rates of new resource requests and in-use resources, respectively. The evaluation results are presented and discussed in the following subsections.

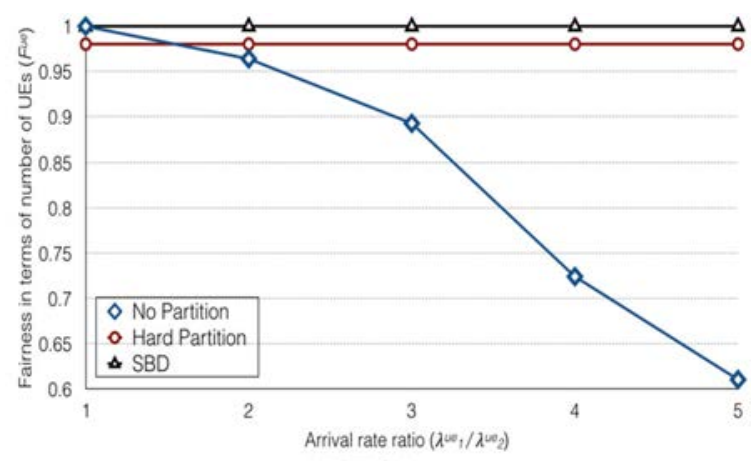

(a) UE fairness

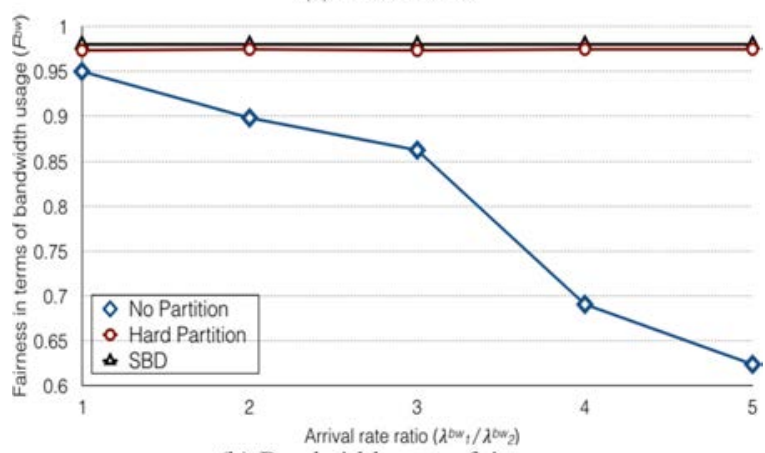

(b) Bandwidth usage fairness

Fig. 9. Fairness among multiple operators.

1) Fairness Among Multiple Operators: The fairness of the proposed system was evaluated using (2) and (3). Given the absence of resource control (i.e., no partition), UE arriving first simply attach to the shared BS until the maximum number of served UE is reached. For a hard partition mechanism, each operator can use only the resources allocated to it, and overusing the BS resource is strictly forbidden. With SBD, on the other hand, operators are allowed to overuse the resource, as described in Section IV. Fig. 9 shows the UE fairness (Fig. 9(a)) and bandwidth usage fairness (Fig. 9(b)) of the considered system given the use of the three different resource allocation mechanisms (i.e., no partition, hard partition, and SBD) and different values of the UE arrival rate ratio (subject to the constraints $\sum_{i=1}^{n} \lambda_{i}{ }^{u e}=5$, and $\left.\sum_{i=1}^{n} \lambda_{i}{ }^{b w}=5 M\right)$. As expected, in the no partition scenario, the operator with a higher UE arrival rate consumes more resource, and hence the fairness decreases. However, for the hard partition scheme and SBD, the fairness is close to 1 (perfectly fair) and shows only a small variation with the UE arrival ratio since the shared BS is not fully loaded. Notably, SBD achieves a slightly better performance than the hard partition scheme since the operators are able to inject more UE into the system on some occasions (e.g., the over using operator can increase the fairness rate by (2) and (3)). As a result, SBD achieves an inter-operator fairness of 0.997, which is higher than that of both the hard-partition approach (0.98) and the no-partition approach (0.6).

2) Utilization of Shared BS: The utilization of the shared BS was evaluated using (4) and (5). Fig. 10 shows the utilization of the shared BS in terms of the number of UE (Fig. 10(a)) and bandwidth usage (Fig. 10(b)) for two partition methods (hard partition and SBD) and various values of the UE arrival rate ratio 


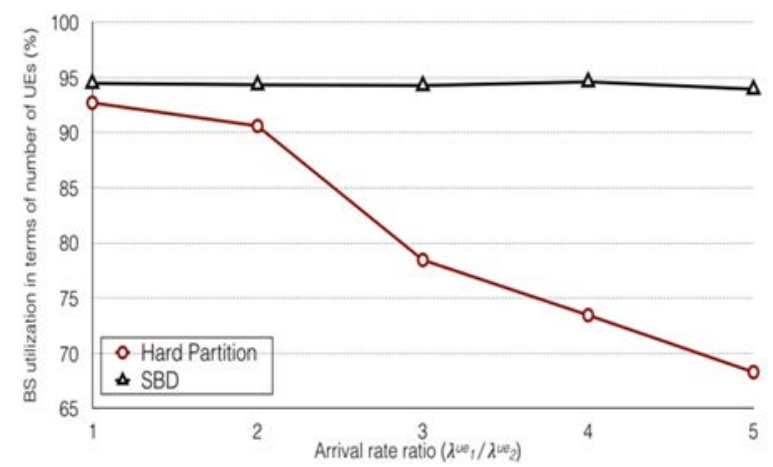

(a) UE utilization rate of shared BS

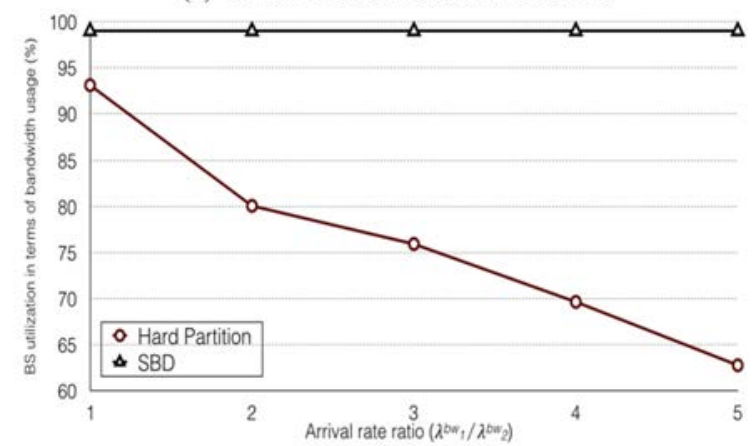

(b) Bandwidth usage rate of shared BS

Fig. 10. Shared base station utilization rate under hard partition and SBD mechanisms.

(subject to the constraints $\sum_{i=1}^{n} \lambda_{i}{ }^{u e}=2$, and $\sum_{i=1}^{n} \lambda_{i}{ }^{b w}=$ $1 M)$. In performing the experiment, $\lambda_{1}^{u e}$ and $\lambda_{1}^{b w}$ were fixed, and $\lambda_{2}^{u e}$ and $\lambda_{2}^{b w}$ were gradually reduced. As shown in Fig. 10(a), the utilization rate achieved by the hard partition scheme is close to that of SBD when the arrival rate is identical. However, the utilization rate drops to $70 \%$ when $\frac{\lambda_{1}^{\mu e}}{\lambda_{2}^{u e}}=5$. By contrast, that of the SBD method remains close to $95 \%$. As shown in Fig. 10(b), SBD improves the bandwidth utilization of the shared BS from $63 \%$ (hard partition) to $98 \%$ when $\frac{\lambda_{1}^{b w}}{\lambda_{2}^{b w}}=5$. The deviation of the utilization rate from $100 \%$ is to be expected since the system does not reside in a fully-loaded condition all of the time.

In general, the results confirm that SBD yields a significant improvement in the bandwidth utilization of the shared BS compared to the hard partition scheme. Consequently, if RANP is operated by a third-party operator, and the revenue is utilizationbased, SBD provides the potential to achieve significant economic benefit.

3) Blocking Rate and Dropping Rate: The blocking rate and dropping rate were computed using (6)-(9). Fig. 11 shows the blocking and dropping rates of the $i$-th operator in terms of the number of UE (Fig. 11(a)) and bandwidth usage (Fig. 11(b)) for two bandwidth assignment mechanisms (hard partition and SBD) and various values of the UE arrival ratio (subject to constraints $\sum_{i=1}^{n} \lambda_{i}{ }^{u e}=2$, and $\sum_{i=1}^{n} \lambda_{i}{ }^{b w}=1 M$ ). In performing the experiments, $\lambda_{1}^{u e}$ and $\lambda_{1}^{b w}$ were fixed and $\lambda_{2}^{u e}$ and $\lambda_{2}^{b w}$ were gradually reduced. In other words, the BS resources (number of served UE and bandwidth) assigned to Operator \#2 were gradually under-utilized as the simulation proceeded. As

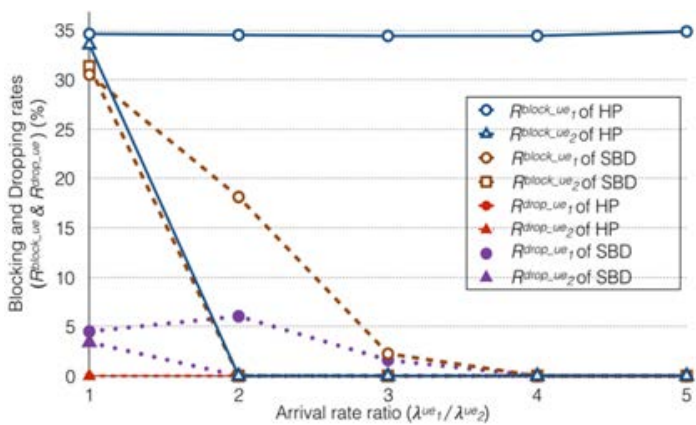

(a) UE blocking and dropping rates

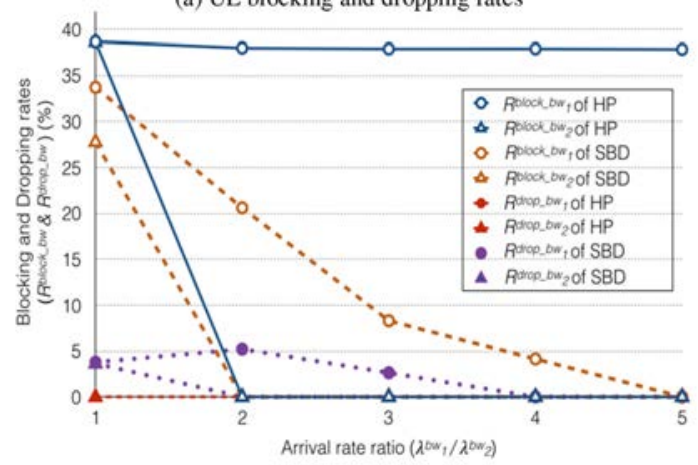

(b) Bandwidth usage blocking and dropping rates

Fig. 11. Blocking and dropping rates of hard partition and SBD mechanisms.

shown in Fig. 11(a), SBD reduces $R_{1}^{\text {block } u \text { e }}$ for Operator \#1 from $35 \%$ (hard partition) to almost $0 \%$ by virtue of the UE dropping feature. For Operator \#2, SBD and the hard partition scheme achieve a similar $R_{2}^{\text {block_ue }}$ performance since the UE requirements of the operator gradually reduce, and hence the resources made available to it at the shared RS are always sufficient to meet its needs. Under the hard partition scheme, no UE are dropped for either Operator \#1 or Operator \#2 since neither operator overuses its assigned resource. By contrast, SBD results in a dropping rate of approximately $5 \%$ for Operator \#1, which implies that the operator borrows resources from Operator \#2 on some occasions and is then required to return these resources at some point later on. In general, the tendency of $R_{1}^{\text {drop-ue }}$ resembles an inverted U-shaped curve as the arrival rate ratio of the operators increases. In particular, for $\frac{\lambda_{1}^{\mu e}}{\lambda_{2}^{u e}}=1$, the overuse condition seldom occurs, and hence $R_{1}^{\text {drop-ue }}$ has a low value. For $\frac{\lambda_{1}^{u e}}{\lambda_{2}^{u e}}=2$, Operator $\# 1$ generally resides in an overuse condition, but Operator \#2 still generates requests. Consequently, SBD frequently triggers Operator \#1 to return its overuse resources, and thus $R_{1}^{\text {drop-ue }}$ increases. However, as the arrival rate of Operator \#2 decreases (i.e., the arrival rate ratio increases), the amount of overuse resources which Operator \#2 should return decreases, and hence $R_{1}^{\text {drop-ue }}$ also decreases. The tendencies of the bandwidth usage blocking rates and dropping rates of the two schemes are very similar to those of the UE blocking and dropping rates, as shown Fig. 11(b).

Overall, the results presented in Fig. 11 show that SBD reduces the blocking rate efficiently when the shared BS is under-utilized, and maintains a dropping rate of around 5\%. 


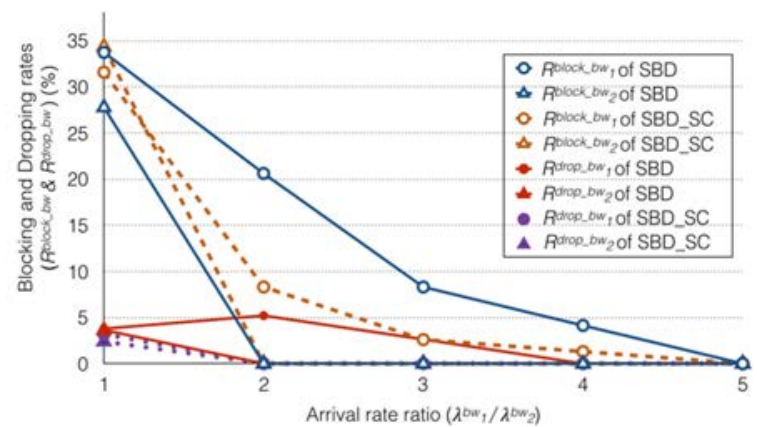

(a) Scale down parameter $=95(\%)$

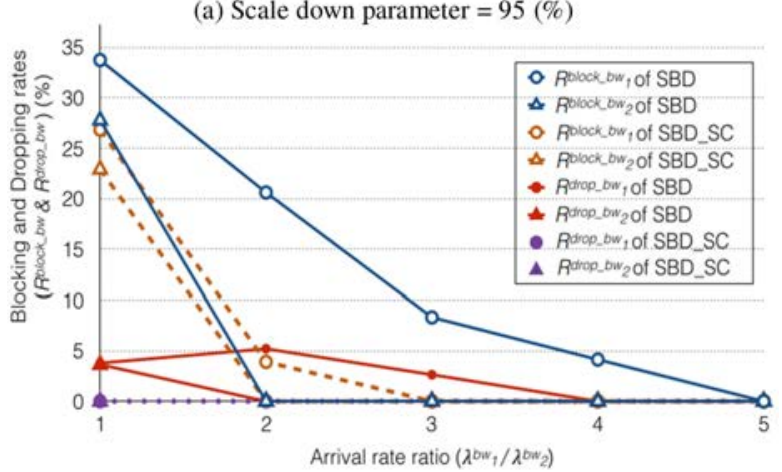

(b) Scale down parameter $=90(\%)$

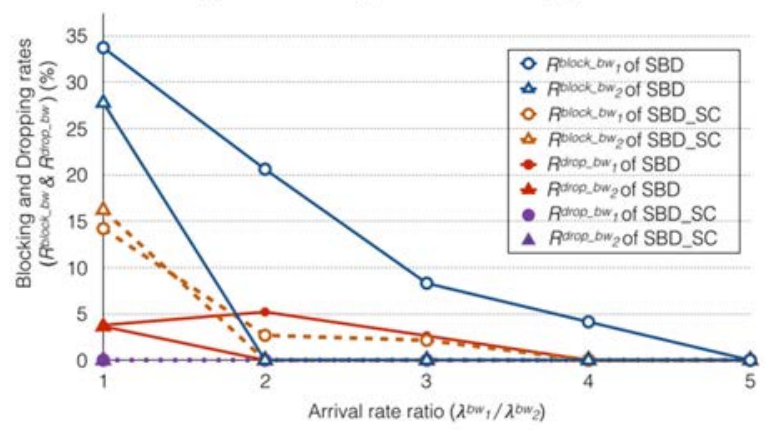

(c) Scale down parameter $=85(\%)$

Fig. 12. Blocking and dropping rates of SBD with and without bandwidth scale-down mechanism.

However, when the shared BS is over-utilized and the two operators have a similar arrival rate, the hard partition method outperforms SBD in terms of a lower dropping rate.

4) SBD With Bandwidth Scale-Down Procedure: The preceding results have shown that SBD improves (i.e., reduces) the blocking rate when the shared BS is under-utilized. However, the dropping rate still remains at around 5\%. Accordingly, further experiments were performed to investigate the performance of the Bandwidth Scale-Down procedure described in Section IV-B in reducing the dropping rate. Fig. 12 shows the SBD bandwidth usage blocking rates and dropping rates for the $i$-th operator with and without the Bandwidth Scale-Down mechanism, respectively, for various values of the UE arrival rate ratio (subject to constraints $\sum_{i=1}^{n} \lambda_{i}{ }^{u e}=2$, and $\sum_{i=1}^{n} \lambda_{i}{ }^{b w}=1 M$.) Note that the experiments were performed using three different values of the scale-down parameter, namely $95 \%, 90 \%$ and $85 \%$. Furthermore, $\lambda_{1}^{u e}$ and $\lambda_{1}^{b w}$ were fixed, but $\lambda_{2}^{u e}$ and $\lambda_{2}^{b w}$ were gradually reduced. In other words, the UE and bandwidth resources assigned to Operator \#2 were progressively underutilized as the simulations proceeded. As shown in Fig. 12(a), $R_{1}^{d r o p \_b w}$ for Operator \#1 reduces from 5\% to almost 3\% when the Bandwidth Scale-Down mechanism is applied (with $s c=95$ ). Furthermore, for Operator $\# 2, R_{2}^{d r o p \_b w}$ reduces from $3 \%$ to $0 \%$. For a slightly lower scale-down value of $s c=90$, $R_{1}^{d r o p \_b w}$ and $R_{2}^{d r o p \_b w}$ both reduce to approximately $0 \%$ when the Bandwidth Scale-Down mechanism is employed (see Fig. 12(b)). A similar result is observed for $s c=85$, as shown in Fig. 12(c). Regarding the blocking rate, Fig. 12(a) shows that for $s c=95$, the blocking rate for Operator \#2, $R_{2}^{\text {block_bd }}$ is very similar regardless of whether or not the Bandwidth Scale-Down mechanism is applied. However, the blocking rate of Operator \#1, $R_{1}^{\text {block_bw }}$, reduces significantly for $\frac{\lambda_{1}^{b w}}{\lambda_{2}^{b w}}>1$. For lower values of the blocking parameter, $R_{1}^{\text {block_bw }}$ and $R_{2}^{\text {block bw }}$ both decrease under the Bandwidth Scale-Down scheme since the amount of bandwidth released may exceed that which was actually requested. In particular, the blocking rate is reduced by approximately $10 \%$ for $s c=90$ and $20 \%$ for $s c=85$ when $\frac{\lambda_{1}^{b w}}{\lambda_{2}^{b w}}=1$. However, no further reduction is obtained for $\frac{\lambda_{1}^{b w}}{\lambda_{2}^{b w}}>1$. Overall, the results confirm the effectiveness of the proposed Bandwidth Scale-Down mechanism in reducing both the dropping rate and the blocking rate provided that $s c$ is properly assigned.

\section{CONCLUSIONS AND Future WORK}

A Soft-partition with Blocking and Dropping (SBD) protocol has been proposed for controlling the fairness among different operators in transparent RAN sharing. In the proposed approach, telecom operators are permitted to utilize more resources than specified in the original Inter-Operator Agreement when the shared BS is under-utilized. However, SBD continuously monitors the usage of the resources for each involved operator and dynamically adjusts the available resources among the operators using blocking or even dropping mechanisms. The simulation results have shown that SBD not only ensures fairness among the operators, but also maintain the utilization rate of the shared BS at almost $100 \%$. Thus, SBD offers the potential for significant economic benefit if the revenue of the BS is utilization-based. Furthermore, SBD reduces the blocking rate from $35 \%$ under a hard partition scheme to almost $0 \%$ when the shared $\mathrm{BS}$ is under-utilized, while maintaining a dropping rate of approximately $5 \%$. Notably, the blocking and dropping rates of SBD can both be improved by using a Bandwidth Scale-Down algorithm with an appropriate scale-down setting.

Future studies will address three main issues. (1) An authenticated-API will be designed for SBD with Bandwidth Scale-Down to permit SBD to obtain UE-realted information from the $\mathrm{CN}$ and send ERAB-Messages to modify bearers in a secure fashion. (2) The practical feasibility of migrating SBD with Bandwidth Scale-Down to $5 \mathrm{G}$ systems will be investigated by, for example, setting up RANP in the Mobile Edge Computing (MEC) [19]-[21] architecture, or running RANP with SBD 
as a third-party service in the C-RAN [22]-[24] architecture with mechanisms designed to avoid block malicious services in between. (3) A comprehensive model will be developed to analyze the effectiveness of SBD in terms of the resource utilization, blocking rate and dropping rate in order to further evaluate the performance of SBD under different resource sharing policies, network loads, UE traffic patterns, and so on.

\section{REFERENCES}

[1] T. Frisanco, P. Tafertshofer, P. Lurin, and R. Ang, "Infrastructure sharing and shared operations for mobile network operators From a deployment and operations view," in Proc. IEEE Netw. Oper. Manage. Symp., Apr. 2008, pp. 129-136.

[2] GSMA Head Office, London, U.K., "Mobile infrastructure sharing," White Paper, Sep. 2012.

[3] Nokia Sol. Netw., Espoo, Finland, "Network sharing: delivering mobile broadband more efficiently and at lower cost," White Paper, 2014.

[4] Accedian Netw., Montreal, QC, Canada, "RAN sharing solutions: Network performance monitoring," White Paper, 2015.

[5] J. Markendahl and A. Ghanbari, "Shared small cell networks multioperator or third party solutions or both?" in Proc. Model. Optim. Mobile, Ad Hoc Wireless Netw., May 2013, pp. 41-48.

[6] Y. D. Lin, H. T. Chien, H. W. Chang, C.-L. Lai, and K.-Y. Lin, "Transparent RAN sharing of $5 \mathrm{G}$ small and macro cells," IEEE Wireless Commun. Mag., vol. 24, no. 6, pp. 104-111, Dec. 2017.

[7] 3GPP, Sophia Antipolis, France, "Radio resource control," 3GPP TS 36.331 V10.7.0, Nov. 2012

[8] L. Frenzel, "Understanding the small-cell and hetnet movement," Electron. Des., vol. 61, Sep. 2013, Art. no. 48.

[9] X. Ge, S. Tu, G. Mao, C.-X. Wang, and T. Han, "5G ultra-dense cellular networks," IEEE Wireless Commun., vol. 23, no. 1, pp. 72-79, Feb. 2016.

[10] 3GPP, Sophia Antipolis, France, "Quality of service (QoS) concept and architecture," 3GPP Specification, TS 23.107 V10.1.0, Jun. 2011.

[11] 3GPP, Sophia Antipolis, France, "Policy and charging control architecture," 3GPP Specification, TS 23.203 V10.10.0, Dec. 2014.

[12] H. Ekström, "QoS control in the 3GPP evolved packet system," IEEE Commun. Mag., vol. 47, no. 2, pp. 76-83, Feb. 2009.

[13] 3GPP, Sophia Antipolis, France, "General packet radio service (GPRS) enhancements for evolved universal terrestrial radio access network (E-UTRAN) access," 3GPP Specification, TS 23.401 V10.13.0, Dec. 2014.

[14] 3GPP, Sophia Antipolis, France, "General packet radio service (GPRS); service description," 3GPP Specification, TS 23.060 V10.3.0, Mar. 2011.

[15] K. Johansson, M. Kristensson, and U. Schwarz, "Radio resource management in roaming based multi-operator WCDMA networks," in Proc. IEEE 59th Veh. Technol. Conf., May 2004, pp. 2062-2066.

[16] A. Gogic and G. B. Horn, "Asymmetric radio access network (RAN) resource allocation in RAN sharing arrangement," U.S. patent 20140029 529, Jan. 30, 2014.

[17] T. Guo and R. Arnott, "Active LTE RAN sharing with partial resource reservation," in Proc. IEEE 78th Veh. Technol. Conf., Sep. 2013, pp. 1-5.

[18] 3GPP, Sophia Antipolis, France, "S1 application protocol," 3GPP Specification, TS 36.413 V10.5.0, Mar. 2012.

[19] ETSI, Sophia Antipolis, France, "Mobile edge computing (MEC) terminology," ETSI Specification, GS MEC, 001 V1.1.1, Mar. 2016.

[20] Y. C. Hu, M. Patel, D. Sabella, N. Sprecher, and V. Young, "Mobile edge computing a key technology towards 5G," ETSI, Sophia Antipolis, France, White Paper, Sep. 2015.

[21] A. Ahmed and E. Ahmed, "A survey on mobile edge computing," in Proc. Int. Conf. Intell. Syst. Control, Jan. 2016, pp. 1-8.

[22] X. Costa-Perez, J. Swetina, and T. Guo, "Radio access network virtualization for future mobile carrier networks," IEEE Commun. Mag., vol. 51, no. 7, pp. 27-35, Jul. 2013.

[23] A. Checko et al., "Cloud RAN for mobile networks - A technology overview," IEEE Commun. Surv. Tut., vol. 17, no. 1, pp. 405-426, Jan.Mar. 2015.

[24] M. Peng, Y. Sun, X. Li, Z. Mao, and C. Wang, "Recent advances in cloud radio access networks: system architectures, key techniques, and open issues," IEEE Commun. Surv. Tut., vol. 18, no. 3, pp. 2282-308, Jul.-Sep. 2016.

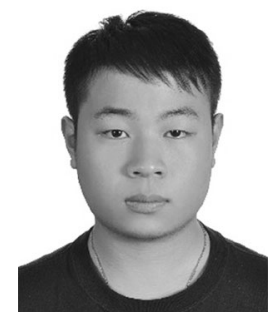

Hsu-Tung Chien received the B.S. and M.S. degrees in computer science from Tung Hai University, Taichung, Taiwan, in 2014, and the M.S. degree in computer science from the National Chiao Tung University (NCTU), Hsinchu, Taiwan in 2017. Since 2017, he was working toward the Ph.D. degree, NCTU. His research interests include wireless networks, mobile networks, and protocol design. $\mathrm{He}$ is also a part of an $\mathrm{H} 2020$ project in 5GPPP, 5GCORAL, to develop the edge and fog systems for $5 \mathrm{G}$ networks.

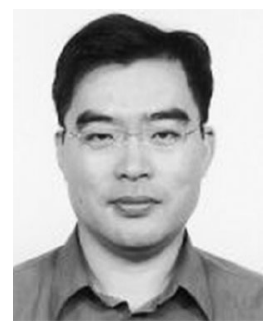

Ying-Dar Lin (F'13) received the Ph.D. degree in computer science from the University of California, Los Angeles, Los Angeles, CA, USA, in 1993. He is currently a Distinguished Professor of computer science with the National Chiao Tung University, Hsinchu, Taiwan. He was a Visiting Scholar with the Cisco Systems, San Jose, CA, USA, during 2007-2008, the CEO with the Telecom Technology Center, Taiwan, during 2010-2011, and the Vice-President of National Applied Research Laboratories, Taiwan, during 2017-2018. Since 2002, he has been the founder and the Director of the Network Benchmarking Laboratory (www.nbl.org.tw), which reviews network products with real traffic and automated tools, and has been an approved test laboratory of the Open Networking Foundation since July 2014. He also cofounded L7 Networks, Inc., in 2002, later acquired by the D-Link Corporation, and O.Prueba, Inc., in 2018 He has coauthored a textbook Computer Networks: An Open Source Approach (McGraw-Hill, 2011) (www.mhhe.com/lin), with Ren-Hung Hwang and Fred Baker. His work on multi-hop cellular was the first along this line, and has been cited more than 850 times and standardized into IEEE 802.11s, IEEE 802.15.5, IEEE 802.16j, and 3GPP LTE-Advanced. His research interests include network security, wireless communications, and network softwarization. He was an IEEE Distinguished Lecturer during 2014-2017 and the ONF Research Associate. He was the recipient of the Research Excellence Award in 2017 and the K. T. Li Breakthrough Award. He has served or is serving on the editorial boards of several IEEE journals and magazines, and is the Editor-in-Chief for the IEEE COMMUNICATIONS SURVEYS AND TUTORIALS.

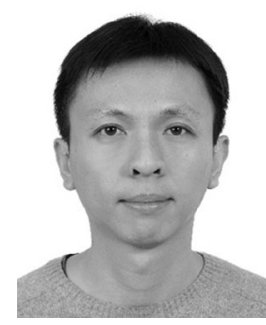

Hsien-Wen Chang received the B.S. and M.S. degrees in electrical engineering from National Tsing Hua University, Hsinchu, Taiwan, in 1999 and 2001, respectively, and the Ph.D. degree in communications engineering from National Chiao Tung University, Hsinchu, in 2015 . He is currently a Senior Engineer with the Industrial Technology Research Institute (ITRI), Hsinchu. From 2001 to 2005, he was with the National Space Organization. Since 2006, he has been with the Information and Communications Research Laboratories, ITRI. His research interests include wireless digital communications, physical-layer and crosslayer algorithms for $5 \mathrm{G}$ new radio transceiver design, and signal processing for massive-MIMO systems.

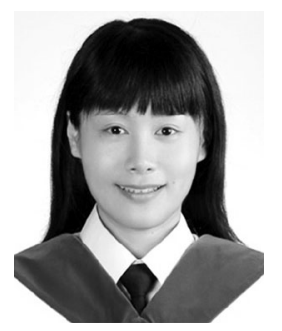

Chia-Lin Lai received the M.S. and Ph.D. degrees from National Cheng-Kung University, Tainan, Taiwan, in 2008 and 2014, respectively. She is currently with the Information and Communications Research Laboratories, Industrial Technology Research Institute, Hsinchu, Taiwan. Her research interests include optical networks, integrated fiber-wireless networks, next generation network architecture, and protocol design. She is a delegate to 3GPP SA Working Group (WG) 2 and focuses on related SIDs and WIDs of $5 \mathrm{G}$ network topics currently running in 3GPP. She is also a part of the H2020 project in 5GPPP; 5G-Transformer to develop the transport network architecture and network slicing algorithms for $5 \mathrm{G}$ networks, and holds several patents in the United States, Mainland China, and Taiwan. 